\title{
Three Layers of Neutrinos
}

\author{
Vernon Barger, Pavel Fileviez Pérez, and Sogee Spinner \\ Department of Physics, University of Wisconsin, Madison, WI 53706, USA
}

(Dated: October 23, 2018)

\begin{abstract}
In this letter we point out that in a class of models for spontaneous R-parity breaking based on gauged B-L, the spectrum for neutrinos is quite peculiar. We find that those models generally predict three layers of neutrinos: one heavy sterile neutrino, two massive active neutrinos, and three nearly massless (one active and two sterile) neutrinos.
\end{abstract}

Introduction: The existence of massive neutrinos have motivated an infinite number of studies in physics beyond the standard model. Today, we know with good precision the values of the mass squared differences and mixing angles: $\Delta m_{12}^{2}=(7.58 \pm 0.21) \times 10^{-5} \mathrm{eV}^{2},\left|\Delta m_{23}^{2}\right|=(2.40 \pm$ $0.15) \times 10^{-3} \mathrm{eV}^{2}$ for the solar and atmospheric mass splittings, $\tan ^{2} \theta_{12}=0.484 \pm 0.048, \sin ^{2} 2 \theta_{23}=1.02 \pm 0.04$ for the solar and atmospheric mixing angles, and $\sin ^{2} 2 \theta_{13}=$ $0.07 \pm 0.04$ [1]. Unfortunately, as in the case of the quark sector of the Standard Model (SM), we do not know the underlying theory for neutrino masses which could explain the above values and predict the type of spectrum: Normal Hierarchy (NH), Inverted Hierarchy (IH) or Quasi-Degenerated (QD).

Recently, we have investigated in great detail the different theories where one can understand the origin of R-parity (non)conservation in supersymmetry [2-5]. In this context, we have found that if one sticks to the minimal model, Rparity must be broken spontaneously by the vacuum expectation values of the "right-handed" sneutrinos present in the theory. These theories based on gauged B-L make a great number of predictions relevant for the discovery of supersymmetry at the Large Hadron Collider since the symmetry breaking scale (i.e. the $Z^{\prime}$ mass), and the R-parity breaking scale are defined by the SUSY breaking scale which is at a TeV. Proton decay [6] in these frameworks occur only through higherdimensional operators and the gravitino can be a good dark matter candidate. See Refs. [2-4] for details and for general aspects of R-parity violation in SUSY see Ref. [7]. For an early study of spontaneous R-parity violation see Ref. [8].

In this letter we investigate the predictions for the neutrino spectrum in the class of models mentioned above. We point out that in these models for spontaneous R-parity breaking in supersymmetry, the spectrum for neutrinos is special. We find that these models generally predict three layers of neutrinos: one heavy sterile neutrino, two massive active neutrinos, and three nearly "massless" (one active and two sterile) neutrinos. Therefore, the spectrum can be: $m_{6} \gg m_{2,3} \gg m_{1}, m_{4}, m_{5}$ in the $\mathrm{NH}$ and $m_{6} \gg m_{1,2} \gg m_{3}, m_{4}, m_{5}$ in the IH. Here, $m_{6}$ is the mass of the heavy neutrino with mass around $\mathrm{GeV}$ $\mathrm{TeV}, m_{1,2,3}$ are the masses of the active neutrinos, and $m_{4,5}$ correspond to the masses of the light sterile neutrinos.

Theoretical Framework: It is well-known that the relevant scale for neutrino mass generation through the seesaw mechanism [9] is typically defined by the $B-L$ scale. Here
$\mathrm{B}$ and $\mathrm{L}$ stand for baryon and lepton number, respectively. The global $B-L$ in the Standard Model (SM) is an accidental anomaly free symmetry, and once it is gauged, the $U(1)_{B-L}^{3}$ and $U(1)_{B-L}$ anomalies must be cancelled. This is independent of $B-L$ being embedded in a larger gauge group or combined with the weak hypercharge. The anomaly $B-L$ cancellation conditions can be satisfied by introducing three generations of right-handed neutrinos, which are singlets under the SM.

The Minimal Supersymmetric Standard Model (MSSM) particle content plus 3 right-handed neutrinos have the following $B-L$ charges:

$$
\begin{array}{rlrl}
Q & \sim \frac{1}{3}, \quad u^{c} \sim-\frac{1}{3}, & d^{c} \sim-\frac{1}{3}, \\
L & \sim-1, \quad \nu^{c} \sim 1, \quad e^{c} \sim 1,
\end{array}
$$

while the MSSM Higgses are neutral. Now, if we gauge $B-L$ in this context one can understand the origin of the (non)conservation of R-parity. This symmetry is defined as $R=(-1)^{2 S+3(B-L)}$, where $\mathrm{S}$ is the spin of the particle. Since the crucial phenomenological and cosmological predictions of supersymmetric theories depend of the (non)conservation of this symmetry one should understand this crucial issue.

The breaking of local $B-L$ can be achieved either by introducing new vector-like pairs of Higgses (chiral superfields) with $B-L$ quantum numbers or by considering the minimal particle content [3]. The latter case necessitates a vacuum expectation value (VEV) for the right-handed sneutrinos. Since these have an odd charge, $R$-parity will be spontaneously broken resulting in a theory that has some commonality with bilinear $R$-parity violating models, see Ref. [3]. Then, the nontrivial issue here is to give a large VEV to the right-handed sneutrinos. The solution to this problem was proposed for the first time in Ref. [2], a tachyonic mass term is needed, and later in Ref. [5] we showed how to dynamically generate this mass through the radiative symmetry breaking mechanism.

In order to understand the predictions in the neutrino sector, one must study the symmetry breaking and we proceed by examining the different contributions to the potential in the minimal case. In this context the superpotential is given by [3]

$$
\begin{aligned}
\mathcal{W} & =\mathcal{W}_{\mathrm{MSSM}}+Y_{\nu} L H_{u} \nu^{c}, \\
\mathcal{W}_{\mathrm{MSSM}} & =Y_{u} Q H_{u} u^{c}+Y_{d} Q H_{d} d^{c}+Y_{e} L H_{d} e^{c}+\mu H_{u} H_{d},
\end{aligned}
$$


where the only difference from the MSSM is the addition of a Yukawa coupling between the neutrinos and $H_{u}$. This gives us an $F$-term contribution to the potential which reads as

$$
V_{F} \supset\left|Y_{\nu}\right|^{2}\left|\tilde{\nu}^{c}\right|^{2}\left(\left|H_{u}^{0}\right|^{2}+|\tilde{\nu}|^{2}\right)+\left(-Y_{\nu} \mu^{*} \tilde{\nu} H_{d}^{0 *} \tilde{\nu}^{c}+\text { h.c. }\right) \text {. }
$$

The relevant contribution from the $D$-terms is

$$
V_{D} \supset \frac{1}{8} g_{X}^{2}\left(x_{\nu^{c}}\left|\tilde{\nu}_{i}^{c}\right|^{2}+D_{\mathrm{MSSM}}\right)^{2} .
$$

Here, we have assumed that $B-L$ is a part of a group $U(1)_{X}$, where a particle $\psi$ has a charge $x_{\psi}$. $D_{\text {MSSM }}$ represents contributions from MSSM fields such as left-handed sneutrinos or the Higgses which are neutral under $B-L$ but might still have a nonzero $x$-charge. Finally, the SUSY breaking potential contributes:

$$
V_{\text {Soft }} \supset\left(m_{\tilde{\nu}^{c}}^{2}\right)_{i j} \tilde{\nu}_{i}^{c} \tilde{\nu}_{j}^{c *}+\left(a_{\nu} \tilde{L} H_{u} \tilde{\nu}^{c}+\text { h.c. }\right)+\ldots,
$$

where the soft mass matrix for the right-handed sneutrinos can be diagonalized without loss of generality. We must now consider which are the most important contributions to the scalar potential. The Yukawa coupling of the neutrinos to $H_{u}$ will lead to a neutrino Dirac mass term. Since the only scale in this potential is the SUSY breaking mass scale, any potential seesaw will have a maximum scale of a few $\mathrm{TeV}$. Therefore, $Y_{\nu}$ must still be rather small for neutrino masses, $Y_{\nu} \lesssim 10^{-6}-10^{-5}$, which can be neglected when minimizing for $\tilde{\nu}^{c}$. The VEV of the left-handed sneutrino also contributes to neutrino masses through a seesaw mediated by the gauginos, placing an upper bound around, $\langle\tilde{\nu}\rangle \lesssim 10^{-3} \mathrm{GeV}$, these terms can be ignored as well.

The trilinear $a_{\nu}$ term helps to determine $\langle\tilde{\nu}\rangle$ and so must also be negligible. This leaves the relevant part of the potential as

$$
V=\frac{1}{8} g_{X}^{2}\left(x_{\nu^{c}}\left|\tilde{\nu}_{i}^{c}\right|^{2}+D_{\mathrm{MSSM}}\right)^{2}+\left(m_{\tilde{\nu}^{c}}^{2}\right)_{i}\left|\tilde{\nu}_{i}^{c}\right|^{2} .
$$

Specifically, what we would like to know is how many of the right-handed sneutrinos attain a VEV. Minimizing with respect to $\tilde{\nu}_{j}^{c}$ yields

$$
\frac{1}{4} g_{X}^{2} x_{\nu^{c}} \tilde{\nu}_{j}^{c *}\left(x_{\nu^{c}}\left|\tilde{\nu}_{i}^{c}\right|^{2}+D_{\mathrm{MSSM}}\right)+\left(m_{\tilde{\nu}^{c}}^{2}\right)_{j} \tilde{\nu}_{j}^{c *}=0,
$$

where there is no sum over $j$. At least one non-trivial solution exists if the soft mass squared is negative. Since we have three equations for the same quantity, two options exist: assume that all the soft masses are equal and the three equations become one or return to the case where only one right-handed sneutrino VEV is non-zero. Notice that in the former case, the potential has an $U(3)_{\tilde{\nu}^{c}}$ flavor symmetry, and one can always rotate to a basis where only one of the right-handed sneutrinos acquires a VEV. In general, the other two generations will also get VEVs but these will be smaller than even the VEVs of the left-handed sneutrinos. The upshot of this discussion is that lepton number is only broken in one family and therefore only one of the right-handed neutrinos can get a $\mathrm{TeV}$ scale mass leaving the other two masses to be determined by the parameters responsible for the active neutrino masses. Therefore: the minimal SUSY B - L models or the simplest theories for R-Parity predict the existence of 2 sterile neutrinos which are degenerate or lighter than the active neutrinos.

Neutrino Mass Spectrum: We Continue in the $B-L$ scenario [3] for simplicity. In the basis: $\left(\nu_{i}, \nu_{I}^{c}, \tilde{B}^{\prime}, \tilde{B}, \tilde{W}, \tilde{H}_{d}^{0}, \tilde{H}_{u}^{0}\right)$, the neutralino mass matrix is

$$
\mathcal{M}=\left(\begin{array}{ccccccc}
0_{3 \times 3} & \frac{\left(Y_{\nu}\right)_{j I} v_{u}}{\sqrt{2}} & -\frac{g_{B L} v_{L j}}{2} & -\frac{g_{1} v_{L j}}{2} & \frac{g_{2} v_{L j}}{2} & 0_{3 \times 1} & \frac{\left(Y_{\nu}\right)_{j K} v_{R K}}{\sqrt{2}} \\
\frac{\left(Y_{\nu}\right)_{J i} v_{u}}{\sqrt{2}} & 0_{3 \times 3} & \frac{g_{B L} v_{R J}}{2} & 03 \times 1 & 0_{3 \times 1} & 0_{3 \times 1} & \frac{\left(Y_{\nu}\right)_{k J} v_{L k}}{\sqrt{2}} \\
-\frac{g_{B L} v_{L i}}{2} & \frac{g_{B L} v_{R L}}{2} & M_{B L} & 0 & 0 & 0 & 0 \\
-\frac{g_{1} v_{L i}}{2} & 0_{1 \times 3} & 0 & M_{1} & 0 & -\frac{g_{1} v_{d}}{2} & \frac{g_{1} v_{u}}{2} \\
\frac{g_{2} v_{L i}}{2} & 0_{1 \times 3} & 0 & 0 & M_{2} & \frac{g_{2} v_{d}}{2} & -\frac{g_{2} v_{u}}{2} \\
0 & 0 & 0 & -\frac{g_{1} v_{d}}{2} & \frac{g_{2} v_{d}}{2} & 0 & -\mu \\
\frac{\left(Y_{\nu}\right)_{K i} v_{R K}}{\sqrt{2}} & \frac{\left(Y_{\nu}\right)_{I k} v_{L k}}{\sqrt{2}} & 0 & \frac{g_{1} v_{u}}{2} & -\frac{g_{2} v_{u}}{2} & -\mu & 0
\end{array}\right)
$$

Here,

$$
\begin{aligned}
& v_{R}=\frac{\sqrt{-8 m_{\tilde{\nu}^{c}}^{2}}}{g_{B L}} \\
& v_{L}=\frac{B_{\nu} v_{R}}{\left(m_{\tilde{L}}^{2}-\frac{1}{8} g_{B L}^{2} v_{R}^{2}\right)},
\end{aligned}
$$

with $B_{\nu}=\frac{1}{\sqrt{2}}\left(Y_{\nu} \mu v_{d}-a_{\nu} v_{u}\right)$. Again, $m_{\tilde{\nu}^{c}}^{2}<0$, see Refs. [2, 5]. As discussed above, only one generation of the "right-handed" sneutrinos gets a TeV size VEV, while the rest are quite small and so we proceed with $v_{R 1}=v_{R 2}=0$ and $v_{R} \equiv v_{R 3} \neq 0$. For an early study of the generation of neutrino masses using the VEV of right-handed sneutrinos see Refs. [8, 10]. One can neglect the active neutrinos 
to try to understand how many heavy states exist in $\mathcal{M}$. We know that this number is equal to five (for the neutralinos) plus some number of the right-handed neutrinos. The characteristic polynomial reveals that only one right-handed neutrino attains a large mass, hence verifying the earlier claim that only one generation gets a large Majorana mass. The mass matrix $\mathcal{M}$ then contains six heavy states, which can be integrated out using the seesaw mechanism to approximate the masses of the light neutrinos (three active and two sterile):

$$
\mathcal{M}_{\nu}=m-m_{D} M^{-1} m_{D}^{T}
$$

where the light Majorana mass matrix is given by

$$
m=\left(\begin{array}{cc}
0_{3 \times 3} & \frac{\left(Y_{\nu}\right)_{i \beta} v_{u}}{\sqrt{2}} \\
\frac{\left(Y_{\nu}\right)_{\alpha i} v_{u}}{\sqrt{2}} & 0_{2 \times 2}
\end{array}\right)
$$

Here, $\alpha$ and $\beta$ run over the two light right-handed neutrinos. The heavy Majorana mass matrix is given by

$$
M=\left(\begin{array}{cccccc}
0 & \frac{g_{B L} v_{R}}{2} & 0 & 0 & 0 & \frac{\left(Y_{\nu}\right)_{k 3} v_{L k}}{\sqrt{2}} \\
\frac{g_{B L} v_{R}}{2} & M_{B L} & 0 & 0 & 0 & 0 \\
0 & 0 & M_{1} & 0 & -\frac{g_{1} v_{d}}{2} & \frac{g_{1} v_{u}}{2} \\
0 & 0 & 0 & M_{2} & \frac{g_{2} v_{d}}{2} & -\frac{g_{2} v_{u}}{2} \\
0 & 0 & -\frac{g_{1} v_{d}}{2} & \frac{g_{2} v_{d}}{2} & 0 & -\mu \\
\frac{\left(Y_{\nu}\right)_{3 k} v_{L k}}{\sqrt{2}} & 0 & \frac{g_{1} v_{u}}{2} & -\frac{g_{2} v_{u}}{2} & -\mu & 0
\end{array}\right)
$$

and finally the Dirac mass matrix is

$$
m_{D}=\left(\begin{array}{cccccc}
\frac{\left(Y_{\nu}\right)_{i 3} v_{u}}{\sqrt{2}} & -\frac{g_{B L} v_{L i}}{2} & -\frac{g_{1} v_{L i}}{2} & \frac{g_{2} v_{L i}}{2} & 0_{3 \times 1} & \frac{\left(Y_{\nu}\right)_{i 3} v_{R}}{\sqrt{2}} \\
0_{2 \times 1} & 0_{2 \times 1} & 0_{2 \times 1} & 0_{2 \times 1} & 0_{2 \times 1} & \frac{\left(Y_{\nu}\right)_{k \alpha} v_{L k}}{\sqrt{2}}
\end{array}\right) .
$$

Before studying the specific form of the resulting five-by-five mass matrix, it is useful to examine the matrix in a very general way. For this purpose it can be cast as

$$
\mathcal{M}_{\nu}=\left(\begin{array}{cc}
M^{L L} & M^{L R} \\
M^{L R^{T}} & M^{R R}
\end{array}\right)
$$

where $M^{L L}$ is the three-by-three left-handed Majorana mass matrix, $M^{R R}$ is the two-by-two right-handed Majorana mass matrix and $M^{L R}$ is the Dirac mixing between the left-handed and right-handed sectors. Such a form allows for four possibilities:

1. Mixed: $M^{L R} \sim M^{L L}$ or $M^{R R}$.

2. Pseudo-Dirac: $M^{L R} \gg M^{L L}, M^{R R}$.

3. "Inverted" seesaw: $M^{L L} \gg M^{L R}, M^{R R}$.

4. "Traditional" seesaw $M^{R R} \gg M^{L R}, M^{L L}$.

Case 1 is already ruled out since it would allow for large mixings between active and light sterile neutrinos, which has not been experimentally observed. Case 2 leads to one Majorana left-handed neutrino and two so called pseudo-Dirac neutrinos. The pseudo-Dirac neutrinos have a small mass splitting between the active and sterile neutrinos on the order of magnitude of the Majorana mass and with a near maximal mixing. Because of this, the mass splitting is severally restricted, see for example Ref. [11]. Cases 3 and 4 allows for all active and sterile neutrinos to be Majorana. Here active-sterile mixings will be controlled by the Dirac mass and therefore this quantity will be bounded by data on neutrino mixing. We will see that only Case 3 is possible in our models.

The specific forms of these submatrices are linear combinations of matrices that are made up of products of elements with flavor structure, i.e. Yukawa couplings and sneutrino VEVs, where the coefficients are ratios of gaugino/Higgsino masses. $\mathcal{M}_{\nu}$ can be treated as an expansion in the flavorful parameters, where to leading order we keep terms with a maximum product of two flavorful parameters. Then, one gets the following mass matrix for the light (active and sterile) neutrinos:

$$
M_{\nu}=\left(\begin{array}{cc}
A v_{L i} v_{L j}+B\left[\left(Y_{\nu}\right)_{i 3} v_{L j}+\left(Y_{\nu}\right)_{j 3} v_{L i}\right]+C\left(Y_{\nu}\right)_{i 3}\left(Y_{\nu}\right)_{j 3} & \frac{1}{\sqrt{2}} v_{u}\left(Y_{\nu}\right)_{i \beta} \\
\frac{1}{\sqrt{2}} v_{u}\left(Y_{\nu}\right)_{\alpha j} & D\left[\left(Y_{\nu}\right)_{k \alpha} v_{L k}\right]\left[\left(Y_{\nu}\right)_{m \beta} v_{L m}\right]
\end{array}\right)
$$


where

$$
\begin{gathered}
A=\frac{2 \mu^{2}}{\tilde{m}^{3}}, \quad B=\left(\frac{v_{u}}{\sqrt{2} v_{R}}+\frac{\sqrt{2} \mu v_{d} v_{R}}{\tilde{m}^{3}}\right), \quad C=\left(\frac{2 M_{B L} v_{u}^{2}}{g_{B L}^{2} v_{R}^{2}}+\frac{v_{d}^{2} v_{R}^{2}}{\tilde{m}^{3}}\right), \quad D=\frac{v_{d}^{2}}{\tilde{m}^{3}}, \\
\tilde{m}^{3}=\frac{4\left[\mu v_{u} v_{d}\left(g_{1}^{2} M_{2}+g_{2}^{2} M_{1}\right)-2 M_{1} M_{2} \mu^{2}\right]}{g_{1}^{2} M_{2}+g_{2}^{2} M_{1}} .
\end{gathered}
$$

From the experimental upper limits on active neutrino masses we obtain $\left(Y_{\nu}\right)_{i \alpha} \lesssim 10^{-12}$. This can be compared to $\left(Y_{\nu}\right)_{i 3} \lesssim$ $10^{-5}$, which is less constrained because of the TeV scale seesaw suppression. The active neutrino masses must come from $M^{L L}$, and it is worthwhile to diagonalize that submatrix first. This can be done by rotating $M_{\nu}$ by

$$
V_{1}=\left(\begin{array}{cc}
U_{i j} & 0_{2 \times 3} \\
0_{3 \times 2} & 1_{2 \times 2}
\end{array}\right)
$$

where $U$ is a three-by-three submatrix that diagonalizes $M^{L L}$. It is easy to show that because of the flavor structure of $M^{L L}$, i.e. only two flavor dependent parameters: $v_{L i}$ and $\left(Y_{\nu}\right)_{i 3}$, it has a zero eigenvalue. Then, only the $\mathrm{NH}$ and $\mathrm{IH}$ are allowed. Assuming $M^{R R}=0$, since it is fourth order in the small parameters (indicating case 4 is not possible), yields

$$
V_{1}^{T} M_{\nu} V_{1}=\left(\begin{array}{cc}
M_{D}^{L L} & \frac{1}{\sqrt{2}} v_{u} U^{T} Y_{\nu} \\
\frac{1}{\sqrt{2}} v_{u} Y_{\nu}^{T} U & 0_{2 \times 2}
\end{array}\right),
$$

where $M_{D}^{L L}=\operatorname{Diagonal}\left(0, m_{2}, m_{3}\right)$ and $\operatorname{Diagonal}\left(m_{1}, m_{2}, 0\right)$ for $\mathrm{NH}$ and $\mathrm{IH}$ respectively. Case 2 is not possible here since $M_{i i}^{L L}$ will would lead to large pseudo-Dirac splitting, then we are left with case 3 as stated earlier. The eigenvalues of the above are two massive active neutrinos and three neutrinos (one active and two steriles) with mass around or bellow the active neutrino scale. This proves our main statement: minimal SUSY B $-L$ models or the simplest theories for $R$-Parity imply the existence of 2 sterile neutrinos which are degenerate or lighter than the active neutrinos. The second case is the most natural scenario.

Summary In this letter we have pointed out that in a class of models for spontaneous R-parity breaking in supersymmetry, the spectrum for neutrinos is quite peculiar. We find that these models generally predict three layers of neutrinos: one heavy sterile neutrino, two massive active neutrinos, and three near massless (one active and two sterile) neutrinos. One can have: $m_{6} \gg m_{2,3} \gg m_{1}, m_{4}, m_{5}$ in the NH scenario and $m_{6} \gg m_{1,2} \gg m_{3}, m_{4}, m_{5}$ in the IH case. Here $m_{6}$ is the mass of the heavy neutrino with mass around $\mathrm{GeV}-\mathrm{TeV}$, $m_{1,2,3}$ are the masses of the active neutrinos, and $m_{4,5}$ are the masses of the light sterile neutrinos. We will discuss the implications for colliders and long baseline neutrino experiments elsewhere.

Acknowledgments: The work of V.B., P.F.P., and S.S. is supported in part by the U.S. Department of Energy under grant No. DE-
FG02-95ER40896, and by the Wisconsin Alumni Research Foundation. Note: Ref. [12], which appeared on the same day as this paper, also studies this topic.

[1] A. Strumia and F. Vissani, "Neutrino masses and mixings and..," arXiv:hep-ph/0606054

[2] P. Fileviez Pérez and S. Spinner, "Spontaneous R-Parity Breaking and Left-Right Symmetry,” Phys. Lett. B 673 (2009) 251; [arXiv:0811.3424 [hep-ph]].

[3] V. Barger, P. Fileviez Perez and S. Spinner, "Minimal gauged $U(1)_{B-L}$ model with spontaneous R-parity violation," Phys. Rev. Lett. 102, 181802 (2009); [arXiv:0812.3661 [hep-ph]].

[4] P. Fileviez Perez and S. Spinner, "Spontaneous R-Parity Breaking in SUSY Models," Phys. Rev. D 80, 015004 (2009); L. L. Everett, P. Fileviez Perez and S. Spinner, "The Right Side of Tev Scale Spontaneous R-Parity Violation,” Phys. Rev. D 80, 055007 (2009); P. Fileviez Perez and S. Spinner, "TeV Scale Spontaneous R-Parity Violation," arXiv:0909.1841 [hep-ph].

[5] P. Fileviez Perez and S. Spinner, "The Fate of R-Parity," arXiv:1005.4930 [hep-ph].

[6] P. Nath and P. Fileviez Pérez, "Proton stability in grand unified theories, in strings, and in branes," Phys. Rept. 441 (2007) 191; P. Fileviez Pérez, "How large could the R-parity violating couplings be?," J. Phys. G 31 (2005) 1025.

[7] R. Barbier et al., "R-parity violating supersymmetry," Phys. Rept. 420 (2005) 1.

[8] M. J. Hayashi and A. Murayama, "Radiative Breaking Of $S U(2)_{R} \times U(1)_{B-L}$ Gauge Symmetry Induced By Broken $N=1$ Supergravity In A Left-Right Symmetric Model," Phys. Lett. B 153 (1985) 251.

[9] P. Minkowski, "Mu $\rightarrow$ E Gamma At A Rate Of One Out Of 1-Billion Muon Decays?," Phys. Lett. B 67 (1977) 421; T. Yanagida, in Proceedings of the Workshop on the Unified Theory and the Baryon Number in the Universe, eds. O. Sawada et al., p. 95, KEK Report 79-18 (1979); M. Gell-Mann, P. Ramond and R. Slansky, in Supergravity, eds. P. van Nieuwenhuizen et al., (North-Holland, 1979), p. 315; R. N. Mohapatra and G. Senjanović, "Neutrino Mass And Spontaneous Parity Nonconservation,” Phys. Rev. Lett. 44 (1980) 912.

[10] R. N. Mohapatra, "Mechanism for understanding small neutrino mass in superstring theories," Phys. Rev. Lett. 56 (1986) 561.

[11] A. de Gouvea, W. C. Huang and J. Jenkins, "Pseudo-Dirac Neutrinos in the New Standard Model," Phys. Rev. D 80, 073007 (2009)

[12] D. K. Ghosh, G. Senjanović and Y. Zhang, "Naturally Light Sterile Neutrinos from Theory of R-parity," arXiv:1010.3968 [hep-ph]. 